\title{
The role of platelet function analyzer-20o in predicting perioperative bleeding risk
}

Eun Sang Yu' ${ }^{1}$, Min Ji Jeon ${ }^{1}$, Ka-Won Kang ${ }^{2}$, Byung-Hyun Lee², Eun Joo Kang ${ }^{1}$, Yong Park ${ }^{2}$, Se Ryeon Lee Hwa Jung Sung ${ }^{3}$, Chul Won $\mathrm{Choi}^{1}$, Byung Soo Kim² ${ }^{2}$, and Dae Sik Kim ${ }^{1}$

\begin{abstract}
${ }^{1}$ Division of Hematology-Oncology, Department of Internal Medicine, Korea University Guro Hospital, Seoul; ${ }^{2}$ Division of HematologyOncology, Department of Internal Medicine, Korea University Anam Hospital, Seoul; ${ }^{3}$ Division of Hematology-Oncology, Department of Internal Medicine, Korea University Ansan Hospital, Ansan, Korea
\end{abstract}

\author{
Received: April 2, 2019 \\ Revised : April 16, 2019 \\ Accepted: April 17, 2019 \\ Correspondence to \\ Dae Sik Kim, M.D. \\ Division of Hematology and \\ Oncology, Department of \\ Internal Medicine, Korea \\ University School of Medicine, \\ 148 Gurodong-ro, Guro-gu, \\ Seoul o8308, Korea \\ Tel: +82-2-2626-3062 \\ Fax: $+82-2-2626-2208$ \\ E-mail: kay9801@naver.com \\ https://orcid.org/0000-0001- \\ $8424-8561$
}

Background/Aims: Various preoperative screening tests, such as platelet count, prothrombin time, activated partial thromboplastin time, and bleeding time, have been widely used to evaluate the risk of bleeding during surgery. Use of platelet function analyzer (PFA)-100/200 for assessing platelet function instead of bleeding time is increasing. However, its role in predicting the perioperative risk of bleeding remains controversial.

Methods: Data of 703 patients who underwent surgery under general anesthesia were retrospectively analyzed. Preoperative platelet function was measured using PFA-200 system and the association with intraoperative bleeding was assessed. Additionally, other variables that could affect PFA-20o results were assessed by logistic regression analysis.

Results: Collagen/epinephrine (COL/EPI) test was prolonged in 199/703 (28.3\%) patients (EPI group), while 99/212 (46.7\%) patients showed COL/adenosine diphosphate test abnormalities. Bleeding over $300 \mathrm{~mL}$ during surgery occurred in $14.3 \%$ and $20.1 \%$ of patients in the normal and EPI groups, respectively $(p=0.058)$. In addition, red blood cell transfusion within 72 hours after surgery rate was significantly higher in the EPI group than in the normal group (31.7\% vs. $23.4 \%, p=$ $0.024)$. In multivariate logistic analysis, prolongation closure time with COL/EPI ( $p$ $=0.068$ ) was marginally associated with risk of bleeding during surgery. Furthermore, PFA-200 results were influenced by various factors, such as nonsteroidal anti-inflammatory drug use, blood group, hematocrit, and time of blood collection.

Conclusions: Preoperative PFA-20o test may be helpful in predicting the risk of perioperative bleeding. However, its results should be carefully interpreted because they are affected by several factors.

Keywords: Platelet function analyzer; Bleeding; Surgery; Screening test

\section{INTRODUCTION}

Excessive bleeding is one of the most important complications of surgery. In case of neurosurgery or cardiac surgery, bleeding complications can lead to devastating consequences. Thus, it is very important to evaluate patient's baseline bleeding tendency and to detect impaired hemostasis prior to invasive procedures such as surgery. In fact, it is common to have hemorrhagic complications that lead to a fatal outcome after surgery without proper evaluation of the hemostasis abnormalities. In order to identify patients with impaired hemostasis, 
it is necessary to assess bleeding history and family history and perform physical examination. In addition, various preoperative laboratory test parameters, including platelet count, prothrombin time (PT), activated partial thromboplastin time (aPTT), and bleeding time (BT), can be used to evaluate the risk of bleeding during surgery [1]. However, their role in assessing the risk of bleeding is still controversial and routine preoperative testing is not recommended in patients without a bleeding history $[1,2]$.

Various coagulation screening tests, such as PT and aPTT, are used to identify defect of coagulation pathway. To evaluate the adequacy of platelet function, BT and platelet aggregation tests has been used for many years. However, the use of BT test is gradually decreasing due to several limitations: low sensitivity, high operator dependency, clinical usefulness, and invasiveness [3]. Platelet aggregation assessed by light transmission aggregometry or whole blood aggregometry is much more sensitive methods for detecting platelet dysfunction compared to BT test. However, these tests are relatively complicated, expensive, and time-consuming [4]. Recently, platelet function analyzer (PFA)-10o has been widely used as an alternative to overcome the limitations of prior tests [5-8]. The PFA-100 is a test device capable of measuring platelet adhesion and aggregation function under high shear-stress blood flow conditions comparable to those that occur in vivo [5]. Platelet adhesion and aggregation occur following exposure to platelet agonists, such as epinephrine (EPI)- or adenosine diphosphate (ADP)-coated membranes. As a result, the time until the membrane aperture occlusion (closure time) is measured by PFA-100. The recently introduced PFA-200 is an upgraded version of the PFA-100.

The most potent role of PFA-100/200 is that of a screening tool for detection of primary hemostasis abnormalities, such as von Willebrand disease (vWD) and various platelet disorders [9,10]. In addition, recent studies have shown its utility in monitoring antiplatelet therapy (including aspirin) and desmopressin therapy in both vWD and platelet disorders $[5,11,12]$. The use of PFA-100/200 test is increasing because it is easy to use and has a high sensitivity to detect hemostasis abnormalities [8]. However, its role as a preoperative screening tool to predict the risk of bleeding during invasive procedure, such as surgery, remains controversial. Some studies have reported that PFA-100/200 results were associated with perioperative bleeding risk, while other studies found no correlation between the calculated blood loss and either preoperative or postoperative PFA100 values [13-16]. Another problem of PFA test is that the closure time (CT) of PFA-10o is influenced by several other factors not actually related to bleeding. To date, blood group, time of blood collection, plasma vWF level, citrate concentration, low platelet count, and hematocrit have been reported to affect CT $[8,17]$.

In this study, we aimed to identify the variables that affect the results of PFA-200 test. Furthermore, we estimated the usefulness of PFA-20o test as a preoperative test to predict the risk of bleeding in patients undergoing various types of surgery.

\section{METHODS}

\section{Patients}

Data of 703 patients older than 18 years who underwent surgery under general anesthesia at Korea University Guro Hospital between October 2015 and April 2016 were retrospectively analyzed. Various real-world types of surgery, ranging from simple surgery, such as appendectomy, to cancer surgery or open-heart surgery, were analyzed in this study. Patients with a history of bleeding disorder or bleeding symptoms at the time of preoperative risk evaluation were excluded. Comprehensive chart reviews of patients' demographic information and previous history, including major comorbidities and the use of anticoagulant and non-steroidal anti-inflammatory drugs (NSAID), were assessed. The Korea University Guro Hospital Institutional Review Board approved this study (2019GRo113). Informed consent was waived by the board.

\section{Measurement of PFA and other variables}

The whole blood sample for platelet function analysis was collected in a $3.2 \%$ sodium citrate anticoagulant bottle. After the collection, samples were kept at room temperature $\left(15^{\circ} \mathrm{C}\right.$ to $\left.25^{\circ} \mathrm{C}\right)$ and transferred to the laboratory. Innovance PFA-200 system (Siemens Healthcare Diagnostics Products, Munich, Germany) was used to assess platelet function. The test was performed at least 15 minutes after the collection of samples for blood 
equilibration and up to 5 hours considering the sample stability according to the manufacturer's recommendation [8]. In our institution, platelet count, PT, aPTT, and PFA are tested in all patients scheduled for surgery to determine the risk of bleeding according to the anesthesiologist's policy. In case of the PFA test, COL/EPI test was performed first and the COL/ADP test was additionally confirmed in patients with an abnormal initial EPI test. However, both COL/EPI and COL/ADP tests were performed simultaneously in some patients. The laboratory results of preoperative evaluation, including complete blood count, PT, aPTT, liver function test, renal function test, electrolytes, and blood group, were collected.

\section{Perioperative bleeding risk evaluation}

Included patients were categorized according to the type of surgery. The severities of surgery were classified to major surgery and minor surgery according to the following criteria. Major surgery was defined as an operation in which a body cavity was entered, a mesenchymal barrier was crossed, a facial plane was opened, an organ was removed, or normal anatomy was altered. Minor surgery or procedure was defined as an any operative procedure in which only the skin, mucous membranes, or superficial connective tissue was manipulated, such as an endoscopic procedure, biopsy, incision \& drainage, or similar procedure [18]. In addition, to assess perioperative bleeding complications, we investigated each patient's blood loss volume during surgery recorded in the operation note, hemoglobin $(\mathrm{Hb})$ concentration before and after the surgery, and the amount of red blood cell (RBC) transfusion performed during and 72 hours after surgery.

\section{Statistical analysis}

All the data were statistically analyzed using IBM SPSS version 20 (IBM Co., Armonk, NY, USA). Patient demographics, preoperative laboratory findings, and bleeding outcome of surgery were compared between two groups according to the results of PFA closure time. Independent $t$ test and Mann-Whitney test were used to detect statistical differences between the two groups for continuous variables. Chi-square test or Kruskal-Wallis test was applied for comparisons of categorical variables. To identify predictive factors for bleeding events during surgery, univariate and multivariate logistic regression analyses were performed. Cox proportional hazards regression analysis was conducted using statistically significant variables in univariate analysis $(p<0.1)$. A $p$ values $<0.05$ was considered statistically significant.

\section{RESULTS}

\section{Patients characteristics}

A total of 703 patients who underwent surgery were evaluated in this study. Of them, 223 (31.7\%) patients showed prolongation of PFA CT just before surgery. Baseline characteristics of patients, abnormal results of PFA test (PFA group), and normal results (normal group) are detailed in Table 1. The proportions of younger and male patients were significantly higher in normal group than in PFA group. The proportion of patients with hypertension or cardiovascular disease was significantly higher in the PFA group than in the normal group. In addition, the use of antiplatelet drugs or NSAID were also higher in the PFA group. Table 2 demonstrates preoperative laboratory results of patients. The proportion of patients with $\mathrm{Hb}$ level < $120 \mathrm{~g} / \mathrm{L}$ was higher in the PFA group than in the normal group (41.3\% vs. $29.4 \%$, $p=0.002$ ). Other factors were not significantly different between the two groups. Table 3 demonstrates the types of surgery assessed in this study. No significant difference was found in the proportion of major surgery between the two groups (63.5\% in normal group vs. $62.3 \%$ in prolongation group, $p=0.757$ ). Abdominal surgery (32.3\%) was the most common type of surgery, followed by orthopedic surgery (31.4\%), and neurosurgery (9.7\%). The categories of surgery applied in the two groups of patients were not significantly different $(p=0.939)$.

\section{PFA closure times}

CT prolongation as revealed by COL/EPI test was detected in 199/703 (28.3\%) patients (EPI group), while 99/212 (46.7\%) patients had abnormalities in the COL/ADP test (ADP group). Seventy-five patients (35.4\%) showed CT prolongation by both tests. Median CTs with COL/ EPI test were 135 seconds (range, 35 to 249) in the normal group and more than 300 seconds (range, 250 to more than 300) in the EPI group. The COL/ADP test was performed in 212 patients, with median CTs of 93 
Table 1. Baseline characteristics of included patients

\begin{tabular}{|c|c|c|c|c|}
\hline \multirow{2}{*}{ Characteristic } & \multirow{2}{*}{ Total $(\mathrm{n}=703)$} & \multicolumn{2}{|c|}{ PFA closure time } & \multirow{2}{*}{ p value } \\
\hline & & $\operatorname{Normal}(n=480)$ & Prolongation $(n=223)$ & \\
\hline Age, yr & $60(18-95)$ & $56(18-94)$ & $61(18-95)$ & $<0.001$ \\
\hline Sex, male/female & $360 / 343$ & $260 / 220$ & $100 / 123$ & 0.021 \\
\hline Body mass index, kg/m² & $23.6(14.1-41.9)$ & $23.4(14.1-41.9)$ & $24.1(15.2-35 \cdot 5)$ & 0.189 \\
\hline \multicolumn{5}{|l|}{ Underlying disease, \% } \\
\hline Hepatic disease & $67(9.5)$ & $44(9.2)$ & $23(10.3)$ & 0.630 \\
\hline Hypertension & $201(28.6)$ & $121(25.2)$ & $80(35 \cdot 9)$ & 0.004 \\
\hline Cardiac disease & $61(8.7)$ & $27(5.6)$ & $34(15.2)$ & $<0.001$ \\
\hline Pulmonary disease & $26(3.7)$ & $17(3.5)$ & $9(4 \cdot 0)$ & 0.747 \\
\hline Cerebrovascular disease & $49(7.0)$ & $29(6.0)$ & $20(9.0)$ & 0.156 \\
\hline Malignancy & $83(11.8)$ & $62(12.9)$ & $21(9.4)$ & 0.181 \\
\hline Diabetes mellitus & $91(12.9)$ & $55(11.5)$ & $36(16.1)$ & 0.085 \\
\hline Thyroid disease & $50(7 \cdot 1)$ & $30(6.2)$ & $20(9.0)$ & 0.192 \\
\hline Chronic kidney disease & $30(4 \cdot 3)$ & $19(4.0)$ & $11(4.9)$ & $0.55^{2}$ \\
\hline Anticoagulant, \% & & & & $<0.001$ \\
\hline Aspirin & $60(8.5)$ & $24(5.0)$ & $36(16.1)$ & \\
\hline Other antiplatelet & $41(5.8)$ & $26(5 \cdot 4)$ & $15(6.7)$ & \\
\hline Aspirin + other & $14(2.0)$ & $5(1.0)$ & $9(4 \cdot 0)$ & \\
\hline NSAID, $\%$ & $252(35.8)$ & $134(27 \cdot 9)$ & $118(52.9)$ & $<0.001$ \\
\hline
\end{tabular}

Values are presented as median (range) or number (\%).

PFA, platelet function analyzer; NSAID, nonsteroidal anti-inflammatory drug.

seconds (range, 43 to 110 ) in the normal group and 138 seconds (range, 110 to 300 ) in the ADP group. Of the factors affecting the results of PFA test, medication history, such as antiplatelet or NSAID, was the most significant. The CT results with COL/EPI of patients taking aspirin (250 seconds) were found to be significantly longer than those patients who did not take anti-platelet drugs (154 seconds) or other anticoagulants (177 seconds) $(p<$ o.001) (Table 4). In case of NSAID, median CT results with COL/EPI were $215 \mathrm{sec}$ for patients who received NSAID, and $147 \mathrm{sec}$ for patients who had no medication $(p<0.001)$. However, the CT results of the COL/ADP test were not affected by antiplatelet $(p=0.391)$ or $\operatorname{NSAID}(p=$ 0.249) drug use. Patients with low Hb level (<120 g/L) in preoperative test showed prolonged CT results in both COL/EPI $(p=0.027)$ and COL/ADP test $(p<0.001)$. Similar results were obtained in patients with low hematocrit $(<35 \%)$. PT and aPTT test were excluded from the anal- ysis because the number of patients who had abnormalities in the preoperative examination was too small. CT results with COL/EPI test were found to be influenced by blood sampling time, with the lowest (148 seconds) in the morning (6:00 to 12:00) and the most prolonged (186 seconds) at night (18:00 to 24:00). CT prolongation in patients with type $\mathrm{O}$ blood group was revealed only by the COL/ADP test $(p=0.022)$ and not by the COL/EPI test $(p=0.916)$.

\section{Bleeding outcomes with surgery}

To confirm the effect of the abnormal PFA results on the operation, several parameters, such as the volume of blood loss during operation, amount of RBC transfusion, and amount of $\mathrm{Hb}$ decrease, were assessed. Table 5 demonstrates postoperative bleeding outcomes between two groups. The rate of patients with bleeding over 300 $\mathrm{mL}$ during surgery was $14.3 \%$ in the normal group and 
Table 2. Baseline laboratory findings

\begin{tabular}{|c|c|c|c|c|}
\hline \multirow{2}{*}{ Characteristic } & \multirow{2}{*}{ Total $(\mathrm{n}=703)$} & \multicolumn{2}{|c|}{ PFA closure time } & \multirow{2}{*}{$p$ value } \\
\hline & & $\operatorname{Normal}(n=480)$ & Prolongation $(\mathrm{n}=223)$ & \\
\hline Hemoglobin, g/L & & & & 0.002 \\
\hline$\geq 120$ & $470(66.9)$ & $339(70.6)$ & $131(58.7)$ & \\
\hline$<120$ & $233(33.1)$ & $141(29.4)$ & $92(41.3)$ & \\
\hline Hematocrit, \% & & & & 0.010 \\
\hline$\geq 35$ & $513(73.0)$ & $366(76.2)$ & $147(65.9)$ & \\
\hline$<35$ & $154(21.9)$ & $90(18.8)$ & $64(28.7)$ & \\
\hline WBC count, /L & & & & 0.766 \\
\hline $4.5^{-11} \times 10^{9}$ & $483(68.7)$ & $330(68.8)$ & $153(68.6)$ & \\
\hline$<4.5 \times 10^{9}$ & $34(4.8)$ & $25(5.2)$ & $9(4 \cdot 0)$ & \\
\hline$\geq 11 \times 10^{9}$ & $186(26.5)$ & $125(26.0)$ & $61(27 \cdot 4)$ & \\
\hline PLT count, /L & & & & 0.837 \\
\hline$\geq 150 \times 10^{9}$ & $636(90.5)$ & $435(90.6)$ & $201(90.1)$ & \\
\hline$<150 \times 10^{9}$ & $67(9.5)$ & $45(9.4)$ & $22(9.9)$ & \\
\hline $\mathrm{PT}$ & & & & 0.324 \\
\hline Normal & $692(98.4)$ & $474(98.8)$ & $218(97.8)$ & \\
\hline Prolongation & $11(1.6)$ & $6(1.2)$ & $5(2.2)$ & \\
\hline aPTT & & & & 0.646 \\
\hline Normal & $660(94.3)$ & $45^{2}(94.6)$ & $208(93.7)$ & \\
\hline Prolongation & $40(5 \cdot 7)$ & $26(5 \cdot 4)$ & $14(6.3)$ & \\
\hline Albumin & & & & 0.681 \\
\hline Normal & $619(88.1)$ & $421(87.7)$ & $198(88.8)$ & \\
\hline Abnormal & $84(11.9)$ & $59(12.3)$ & $25(11.2)$ & \\
\hline Bilirubin & & & & 0.768 \\
\hline Normal & $637(90.6)$ & $436(90.8)$ & $201(90.1)$ & \\
\hline Abnormal & $66(9.4)$ & $44(9.2)$ & $22(9.9)$ & \\
\hline Creatinine & & & & 0.319 \\
\hline Normal & $673(95 \cdot 7)$ & $462(96.2)$ & $211(94.6)$ & \\
\hline Abnormal & $30(4 \cdot 3)$ & $18(3.8)$ & $12(5.4)$ & \\
\hline
\end{tabular}

Values are presented as number (\%).

PFA, platelet function analyzer; WBC, white blood cell; PLT, platelet; PT, prothrombin time; aPT'T, activated partial thromboplastin time.

$20.1 \%$ in the EPI group $(p=0.058)$. No significant difference was detected in median volume of blood loss during surgery between normal and EPI groups (600 $\mathrm{mL}$ vs. $500 \mathrm{~mL}, p=0.569$ ). The proportion of patients with RBC transfusions to restore blood loss during surgery was not significantly different between in normal group and EPI group (15.1\% vs. $20.1 \%, p=0.106)$. To confirm the outcomes of delayed bleeding, we compared the amount of $\mathrm{Hb}$ reduction and the amount of $\mathrm{RBC}$ transfusion within 72 hours after surgery. The proportion of patients with a significant decrease in $\mathrm{Hb}$ level of $30 \mathrm{~g} / \mathrm{L}$ or more was not different between the normal and EPI groups (22.2\% vs. $25.6 \%, p=0.335)$. However, the frequency of RBC transfusion was significantly higher in the EPI group than in the normal group $31.7 \%$ vs. $23.4 \%, p=0.024)$. No significant difference in duration of hospital stay ( 11 days vs. 11 days, $p=0.207$ ) was detected between the two groups. In case of COL/ADP test, no 
Table 3. Types of surgery assessed in the study

\begin{tabular}{|c|c|c|c|c|}
\hline \multirow{2}{*}{ Characteristic } & \multirow{2}{*}{ Total $(\mathrm{n}=703)$} & \multicolumn{2}{|c|}{ PFA closure time } & \multirow{2}{*}{$p$ value } \\
\hline & & Normal (n = 480) & Prolongation $(\mathrm{n}=223)$ & \\
\hline Severity of surgery & & & & 0.757 \\
\hline Major $^{\mathrm{a}}$ & $444(63.2)$ & $305(63.5)$ & $139(62.3)$ & \\
\hline Minor $^{b}$ & $259(36.8)$ & $175(36.5)$ & $84(37 \cdot 7)$ & \\
\hline Category of surgery & & & & 0.939 \\
\hline Orthopedic surgery & $221(31.4)$ & $153(31.9)$ & $68(30.5)$ & \\
\hline Abdominal surgery & $227(32.3)$ & $155(32.3)$ & $72(32.3)$ & \\
\hline Neurosurgery & $68(9.7)$ & $41(8.5)$ & $27(12.1)$ & \\
\hline Thoracic surgery & $44(6.3)$ & $34(7 \cdot 1)$ & $10(4 \cdot 5)$ & \\
\hline Plastic surgery & $26(3.7)$ & $18(3.8)$ & $8(3 \cdot 6)$ & \\
\hline Gynecological surgery & $25(3.6)$ & $16(3 \cdot 3)$ & $9(4 \cdot 0)$ & \\
\hline Otolaryngologic surgery & $17(2.4)$ & $12(2.5)$ & $5(2.2)$ & \\
\hline Urologic surgery & $13(1.8)$ & $9(1.9)$ & $4(1.8)$ & \\
\hline Cardiovascular surgery & $11(1.6)$ & $7(1.5)$ & $4(1.8)$ & \\
\hline Ophthalmic surgery & $4(0.6)$ & $2(0.4)$ & $2(0.9)$ & \\
\hline Breast surgery & $3(0.4)$ & $2(0.4)$ & $1(0.4)$ & \\
\hline Others ${ }^{c}$ & $44(6.3)$ & $31(6.5)$ & $13(5.8)$ & \\
\hline
\end{tabular}

Values are presented as number (\%).

PFA, platelet function analyzer.

${ }^{a}$ Major surgery: any procedure in which a body cavity was entered, a mesenchymal barrier was crossed, a facial plane was opened, an organ was removed, or normal anatomy was altered.

${ }^{\mathrm{b}}$ Minor surgery: any operative procedure in which only the skin, mucous membranes, or superficial connective tissue were manipulated, such as gastroscopy, colonoscopy, and similar procedures.

${ }^{\mathrm{c}}$ Others include biopsy, simple incision \& drainage, catheter insertion, and endoscopic procedure.

significant differences were found between the normal group and ADP group in all of the above variables.

Age (hazard ratio [HR], 1.02; 95\% confidence interval [CI], 1.01 to $1.03 ; p=0.002)$, severity of surgery $(\mathrm{HR}, 3.56$; 95\% CI, 2.04 to 6.22; $p<0.001)$, preoperative aPTT (HR, 2.81; $95 \%$ CI, 1.31 to $6.01 ; p=0.008)$, and serum albumin level (HR, 4.29; 95\% CI; 2.49 to 7.40; $p<0.001$ ) were identified as predictive factors affecting bleeding events during surgery in multivariate logistic analysis (Table 6). CT prolongation in COL/EPI test (HR, 1.55; 95\% CI, 0.97 to $2.47 ; p=0.068$ ) was marginally associated with increased risk of bleeding during surgery. To exclude the confounding effects of anti-platelet drugs and NSAID, subgroup analysis was performed only on patients who did not receive anti-platelets and NSAID. In this subgroup, multivariate logistic analysis revealed that sever- ity of surgery (HR, 3.82; 95\% CI, 1.74 to $8.37 ; p=0.001$ ), preoperative aPTT (HR, 3.29; 95\% CI, 1.05 to $10.32 ; p=$ 0.041), and serum albumin level (HR, 8.03; 95\% CI, 3.70 to $17.45 ; p<0.001$ ) were predictive factors for bleeding. In addition, COL/EPI test prolongation was found to be a significant predictive factor for intraoperative bleeding (HR, 1.16; 95\% CI, 2.62 to $5.93 ; p=0.021$ ).

\section{DISCUSSION}

Unselected preoperative screening tests, such as PT, aPT'T, and PFA-100/200, are generally not recommended for all patients without bleeding history because they may delay surgery inappropriately and cause unnecessary increases in medical costs $[2,8]$. However, unselect- 
Table 4. Factors affecting the results of PFA closure time

\begin{tabular}{|c|c|c|c|c|c|c|}
\hline \multirow{2}{*}{ Characteristic } & \multicolumn{3}{|c|}{ COL/EPI } & \multicolumn{3}{|c|}{ COL/ADP } \\
\hline & Number & Closure time & $p$ value & Number & Closure time & $p$ value \\
\hline \multicolumn{7}{|l|}{ Anti-platelet drug } \\
\hline No use & 587 & $154(35-300)$ & $<0.001$ & 153 & $108(43-300)$ & 0.391 \\
\hline Aspirin & 59 & $250(83-300)$ & & 30 & $118(46-300)$ & \\
\hline Other antiplatelet & 40 & $177(86-300)$ & & 18 & $105(81-300)$ & \\
\hline Combination & 14 & $265(109-300)$ & & 8 & $114(60-300)$ & \\
\hline \multicolumn{7}{|l|}{ NSAID } \\
\hline No & 449 & $147(35-300)$ & $<0.001$ & 111 & $109(43-300)$ & 0.249 \\
\hline Yes & 251 & $215(50-300)$ & & 98 & $107(46-300)$ & \\
\hline \multicolumn{7}{|l|}{ Hemoglobin, g/L } \\
\hline$\geq 120$ & 469 & $153(56-300)$ & 0.027 & 126 & $103(43-300)$ & $<0.001$ \\
\hline$<120$ & 231 & $181(35-300)$ & & 83 & $124(60-300)$ & \\
\hline \multicolumn{7}{|l|}{ Hematocrit, \% } \\
\hline$\geq 35$ & 512 & $157(56-300)$ & 0.039 & 141 & $103(43-300)$ & $<0.001$ \\
\hline$<35$ & 188 & $191(35-300)$ & & 68 & $131(60-300)$ & \\
\hline \multicolumn{7}{|l|}{ Platelet count, /L } \\
\hline$\geq 150 \times 10^{9}$ & 633 & $161(35-300)$ & 0.542 & 191 & $108(43-300)$ & 0.017 \\
\hline$<150 \times 10^{9}$ & 67 & $167(71-300)$ & & 18 & $116(89-300)$ & \\
\hline \multicolumn{7}{|l|}{ Blood collection time $\mathrm{a}^{\mathrm{a}}$} \\
\hline Dawn & 87 & $169(50-300)$ & 0.004 & 36 & $100(60-300)$ & 0.766 \\
\hline Morning & 160 & $148(35-300)$ & & 58 & $107(45-300)$ & \\
\hline Afternoon & 303 & $157(56-300)$ & & 83 & $109(46-300)$ & \\
\hline Night & 150 & $186(81-300)$ & & 35 & $110(43-300)$ & \\
\hline \multicolumn{7}{|l|}{ Blood group } \\
\hline A & 237 & $163(71-300)$ & 0.916 & 71 & $100(46-300)$ & 0.122 \\
\hline $\mathrm{B}$ & 192 & $156(35-300)$ & & 53 & $110(58-300)$ & \\
\hline $\mathrm{AB}$ & 80 & $152(68-300)$ & & 24 & $99(45-300)$ & \\
\hline $\mathrm{O}$ & 191 & $169(50-300)$ & & 61 & $115(43-300)$ & \\
\hline
\end{tabular}

Values are presented as median (range). Prothrombin time and activated partial thromboplastin time were excluded because the number of abnormal results was too small.

PFA, platelet function analyzer; COL/EPI, collagen/epinephrine; COL/ADP, collagen/adenosine diphosphate; NSAID, nonsteroidal anti-inflammatory drug.

${ }^{a}$ Dawn, o:oo-6:0o; Morning, 6:00-12:00; Afternoon, 12:00-18:00; Night, 18:00-24:00.

ed preoperative screening tests are widely used to identify the risk of intraoperative bleeding in real clinical practice. In this study, we aimed to clarify the role of a preoperative PFA-200 test in a relatively large cohort of patients who underwent various types of surgery. As a result, the preoperative CT prolongation revealed by COL/EPI test was associated with increased risk of intra- operative bleeding and postoperative RBC transfusion.

Preoperative screening tests to identify patients with primary or secondary hemostatic disorder is very important because intraoperative bleeding in these patients can cause fatal outcomes. However, until now, it is impossible to predict these diseases only by application of specific tests, so the bleeding history, family 
Table 5. Postoperative bleeding outcomes according to PFA test results

\begin{tabular}{|c|c|c|c|}
\hline \multirow{2}{*}{ Characteristic } & \multicolumn{2}{|c|}{ PFA closure time (COL/EPI) } & \multirow{2}{*}{$p$ value } \\
\hline & Normal & Prolongation & \\
\hline \multicolumn{4}{|l|}{ Intra-operation } \\
\hline Blood loss $\geq 300 \mathrm{~mL}$ & $72(14 \cdot 3)$ & $40(20.1)$ & 0.058 \\
\hline Volume of bleeding & $600(50-5,000)$ & $500(120-6,500)$ & 0.569 \\
\hline RBC transfusion & $76(15.1)$ & $40(20.1)$ & 0.106 \\
\hline \multicolumn{4}{|l|}{ Within 72 hours after surgery } \\
\hline Postoperative $\mathrm{Hb}$ reduction $\geq 30 \mathrm{~g} / \mathrm{L}$ & $112(22.2)$ & $51(25 \cdot 6)$ & 0.335 \\
\hline Postoperative RBC transfusion & $118(23.4)$ & $63(31.7)$ & 0.024 \\
\hline Hospitalization days & $11(1-125)$ & $11(1-159)$ & 0.693 \\
\hline
\end{tabular}

Values are presented as number (\%) or median (range).

PFA, platelet function analyzer; COL/EPI, collagen/epinephrine; RBC, red blood cell; Hb, hemoglobin.

Table 6. Results of logistic regression analysis for intra-operation bleeding loss

\begin{tabular}{|c|c|c|c|}
\hline \multirow{2}{*}{ Factor } & \multirow{2}{*}{$\begin{array}{c}\text { Univariate analysis } \\
\text { p value }\end{array}$} & \multicolumn{2}{|c|}{ Multivariate analysis } \\
\hline & & $\operatorname{HR}(95 \% \mathrm{CI})$ & $p$ value \\
\hline Sex & 0.780 & & \\
\hline Age & $<0.001$ & $1.02(1.01-1.03)$ & 0.002 \\
\hline Severity of surgery & $<0.001$ & $3.56(2.04-6.22)$ & $<0.001$ \\
\hline $\mathrm{BMI}$ & 0.236 & & \\
\hline Use of anticoagulant & $<0.001$ & & \\
\hline Use of NSAID & 0.126 & & \\
\hline Hemoglobin & $<0.001$ & & \\
\hline WBC count & 0.483 & & \\
\hline PLT & 0.064 & & \\
\hline PT & 0.076 & & \\
\hline aPTT & $<0.001$ & $2.81(1.31-6.01)$ & 0.008 \\
\hline Albumin & $<0.001$ & $4.29(2.49-7.40)$ & $<0.001$ \\
\hline Bilirubin & 0.376 & & \\
\hline Creatinine & 0.107 & & \\
\hline COL/EPI & 0.059 & $1.55(0.97-2.47)$ & 0.068 \\
\hline COL/ADP & 0.996 & & \\
\hline
\end{tabular}

HR, hazard ratio; CI, confidence interval; BMI, body mass index; NSAID, nonsteroidal anti-inflammatory drug; WBC, white blood cell; PLT, platelet; PT, prothrombin time; aPT'T, activated partial thromboplastin time; COL/EPI, collagen/epinephrine; COL/ADP, collagen/adenosine diphosphate.

history, and physical examination should be performed. PFA-100 was first introduced in 1995 as a screening test for the diagnosis of vWD and platelet disorders [19]. In addition, the use of PFA-10o instead of BT to assess platelet function has also raised expectations for the role of PFA-100 as a preoperative screening test for primary hemostatic disorders. Koscielny et al. [13] reported in a large cohort study with preoperative questionnaire that the vast majority of patients with impaired hemostasis could be identified by PFA-100. The sensitivity of the PFA-100 COL/EPI CT was the highest (90.8\%) in comparison with the other screening tests $(\mathrm{BT}, \mathrm{aPT} T$, PT, 
vWF:Ag). Fattorutto et al. [14] observed a weak correlation between preoperative COL/EPI CT and second-hour mediastinal blood loss in 70 patients with cardio-pulmonary bypass $(r=0.34, p=0.01)$. However, Lasne et al. [15] and Cammerer et al. [16] reported no correlation between calculated blood loss and either preoperative or postoperative PFA-100 values. The worldwide prevalence of vWD is estimated to be $1 \%$, but in Korea, the number of patients registered in the Korean Hemophilia Foundation by 2017 is only 130, which is much lower than prevalence of this disease in other countries. In case of platelet disorders, such as Bernard-Soulier syndrome and Glanzmann thrombasthenia, the prevalence is difficult to estimate, and only a few cases have been reported. Therefore, it is even more controversial to use PFA-100/200 as a preoperative screening tool for all unselected surgical patients to identify platelet disorders in Korea, where the prevalence of platelet disease is very low. This is consistent with the results of studies reported in Korea that preoperative PFA-10o screening test is less relevant to actual bleeding outcomes [20-22]. However, these studies had limitations to evaluate the role of PFA as a preoperative screening tool in unselected patients because the number of included patients was small and only specific operations were included.

The advantage of our study was that we included a relatively large number of patients and analyzed various types of surgery performed during the study period. Different surgeries may act as confounding factors for the amount of bleeding. However, the effect of variety of surgeries on the amount of blood loss was very small, because the proportion of patients according to the category of surgeries was not different between the two groups even though the category of operation was separately assessed. Another advantage of the present study is that various factors were identified to accurately assess perioperative bleeding. Because of the limitations of retrospective studies, it is difficult to accurately assess the amount of intraoperative bleeding and the amount of delayed blood loss after surgery. We reviewed both the intraoperative anesthesia notes recorded by the anesthesiologist and operation notes written by the surgeon, because surgeons tend to record less blood loss. In addition, the amount of blood transfusion during operation, the amount of transfusion within 72 hours after surgery, and the amount of $\mathrm{Hb}$ decrease within
72 hours after surgery were compared. In our study, PFA-200 prolongation was associated with the rate of intraoperative bleeding and RBC transfusion within 72 hours postoperatively. Although multivariate logistic analysis showed borderline significance, prolonged CT as revealed by COL/EPI was also associated with intraoperative blood loss (HR, 1.55; 95\% CI, 0.97 to 2.47; $p=$ o.068). In addition, these results were more prominent in the subgroup analysis of patients without medication history in whom the bias related to antiplatelet drugs and NSAID use was excluded (HR, 1.16; 95\% CI, 2.62 to 5.93; $p=0.021$ ). These results suggest that preoperative PFA abnormality may increase the risk of perioperative bleeding regardless of the use of antiplatelet agents or NSAID. Therefore, in patients with these abnormalities, accurate history and physical examination and laboratory test findings should be checked to exclude primary hemostatic disorders.

It is widely known that the use of aspirin and NSAID can affect the results of PFA-10o. In addition, it has been reported that the blood group, hematocrit, platelet count, and time of blood collection can affect the results of PFA-100 [8,23-25]. In our study, the use of aspirin and NSAID only affected the results of COL/EPI and did not affect the results of COL/ADP. Low $\mathrm{Hb}$ and hematocrit levels also affected the results of both COL/EPI and COL/ADP tests, which is consistent with the results of previous studies. Platelets count only affected the result of COL/ADP, which is difficult to interpret precisely because the number of patients with low platelet counts included in our study was too small. In particular, since our study was performed on patients who underwent surgery, most patients had platelets counts $>100 \times 10^{9} / \mathrm{L}$ and very few patients had platelet counts $<100 \times 10^{9} / \mathrm{L}$. It has been reported that time of blood collection also affects the results of PFA-100 [17,24]. In accordance with the results of previous results, our study showed that the fast CT of COL/EPI occurred in the morning and the CT results became prolonged during the day. The results of COL/ADP showed a similar tendency, but without statistical significance, which is thought to be because of a diurnal variation in platelet function, which is more dependent on epinephrine than on ADP [26]. In addition, the increased platelet aggregation is estimated to be because of the higher in vivo epinephrine level in the morning, which induces platelet activation [27]. It was 
reported that the CT of PFA-10o was prolonged in the blood group O compared to other blood groups. Our study also showed that the PFA-20o results of group O were the most prolonged, but the statistical significance was not confirmed. Previous studies have shown that CT of PFA-10o is prolonged in the order of group AB, group $\mathrm{B}$, group $\mathrm{A}$, and group $\mathrm{O}$, and our results are also exactly the same $[25,28,29]$. However, the association between blood group and PFA results did not have statistical significance, as the difference between the results of group A and group $\mathrm{O}$ was small. The prolongation of PFA in blood group $\mathrm{O}$ can be explained by the fact that the CT of PFA-100/200 is influenced by the plasma level of $\mathrm{vWF}$ and the vWF concentration, which is lower in blood group $\mathrm{O}$.

In conclusion, preoperative PFA-20o test may be helpful in predicting the risk of bleeding in patients with surgery. However, the results of PFA-200 is influenced by various factors, such as use of aspirin and NSAID, blood group, hematocrit, and time of blood collection, the results of PFA-100/200 should be carefully interpreted after precluding the effects of these confounding factors.

\section{KEY MESSAGE}

1. Preoperative platelet function analyzer (PFA)200 test may be helpful in predicting the risk of bleeding in patients with surgery.

2. PFA-200 is influenced by various factors, such as use of aspirin and non-steroidal anti-inflammatory drugs, blood group, hematocrit, and time of blood collection.

\section{Conflict of interest}

No potential conflict of interest relevant to this article was reported.

\section{REFERENCES}

1. Kozek-Langenecker SA, Afshari A, Albaladejo P, et al. Management of severe perioperative bleeding: guidelines from the European Society of Anaesthesiology. Eur J An- aesthesiol 2013;30:270-382.

2. Chee YL, Crawford JC, Watson HG, Greaves M. Guidelines on the assessment of bleeding risk prior to surgery or invasive procedures. British Committee for Standards in Haematology. Br J Haematol 2008;140:496-504.

3. Rodgers RP, Levin J. A critical reappraisal of the bleeding time. Semin Thromb Hemost 1990;16:1-20.

4. Harrison P. Progress in the assessment of platelet function. Br J Haematol 2000;111:733-744.

5. Favaloro EJ. Utility of the PFA-10o for assessing bleeding disorders and monitoring therapy: a review of analytical variables, benefits and limitations. Haemophilia 2001;7:170-179.

6. Harrison P. The role of PFA-10o testing in the investigation and management of haemostatic defects in children and adults. Br J Haematol 2005;130:3-10.

7. Hayward CP, Harrison P, Cattaneo M, Ortel TL, Rao AK; Platelet Physiology Subcommittee of the Scientific and Standardization Committee of the International Society on Thrombosis and Haemostasis. Platelet function analyzer (PFA)-10o closure time in the evaluation of platelet disorders and platelet function. J Thromb Haemost 2006;4:312-319.

8. Favaloro EJ. Clinical utility of the PFA-100. Semin Thromb Hemost 2008;34:709-733.

9. Fressinaud E, Veyradier A, Truchaud F, et al. Screening for von Willebrand disease with a new analyzer using high shear stress: a study of 6o cases. Blood 1998;91:1325-1331.

10. Mammen EF, Comp PC, Gosselin R, et al. PFA-10o system: a new method for assessment of platelet dysfunction. Semin Thromb Hemost 1998;24:195-202.

11. Favaloro EJ. Clinical application of the PFA-100. Curr Opin Hematol 2002;9:407-415.

12. Favaloro EJ. Laboratory monitoring of therapy in von Willebrand disease: efficacy of the PFA-1oo and von Willebrand factor:collagen-binding activity as coupled strategies. Semin Thromb Hemost 2006;32:566-576.

13. Koscielny J, Ziemer S, Radtke H, et al. A practical concept for preoperative identification of patients with impaired primary hemostasis. Clin Appl Thromb Hemost 2004;10:195-204.

14. Fattorutto M, Pradier O, Schmartz D, Ickx B, Barvais L. Does the platelet function analyser (PFA-100) predict blood loss after cardiopulmonary bypass? Br J Anaesth 2003;90:692-693.

15. Lasne D, Fiemeyer A, Chatellier G, Chammas C, Baron 
JF, Aiach M. A study of platelet functions with a new analyzer using high shear stress (PFA 100) in patients undergoing coronary artery bypass graft. Thromb Haemost 2000;84:794-799.

16. Cammerer U, Dietrich W, Rampf T, Braun SL, Richter JA. The predictive value of modified computerized thromboelastography and platelet function analysis for postoperative blood loss in routine cardiac surgery. Anesth Analg 2003;96:51-57.

17. Cho YU, Chi HS, Jang S, Park CJ. Reconfirmation of preanalytical variables and establishment of reference intervals of platelet function analyzer-100 closure times in Korean adults. Korean J Lab Med 2007;27:318-323.

18. Poon MC, d'Oiron R, Zotz RB, et al. The international, prospective Glanzmann Thrombasthenia Registry: treatment and outcomes in surgical intervention. Haematologica 2015;100:1038-1044.

19. Kundu SK, Heilmann EJ, Sio R, Garcia C, Davidson RM, Ostgaard RA. Description of an in vitro platelet function analyzer: PFA-100. Semin Thromb Hemost 1995;21 Suppl 2:106-112.

20. Lee AJ, Kim SG. Utility of preoperative in vitro platelet function tests for predicting bleeding risk in patients undergoing functional endoscopic sinus surgery. J Blood Med 2016;7:235-238.

21. Kim J, Kim Y, Kwon K, Koo S. The role of PFA-10o measurement in preoperative screening in total knee arthroplasty patients with perioperative replacement of cycloo- xygenase-2 inhibitor. Int J Lab Hematol 2015;37:e34-e37.

22. Jeon Y, Lee WI, Kang SY, Kim MH. Limitations of preoperative PFA-200 as a predictor of postoperative blood loss in total knee arthroplasty: according to use of tranexamic acid. Clin Lab 2017;63:1121-1128.

23. Cho YU, Jang S, Park CJ, Chi HS. Variables that affect platelet function analyzer-100 (PFA-100) closure times and establishment of reference intervals in Korean adults. Ann Clin Lab Sci 2008;38:247-253.

24. Dalby MC, Davidson SJ, Burman JF, Davies SW. Diurnal variation in platelet aggregation iwth the PFA-10o platelet function analyser. Platelets 2000;11:320-324.

25. Moeller A, Weippert-Kretschmer M, Prinz H, Kretschmer $\mathrm{V}$. Influence of ABO blood groups on primary hemostasis. Transfusion 2001;41:56-60.

26. Jafri SM, VanRollins M, Ozawa T, Mammen EF, Goldberg $\mathrm{AD}$, Goldstein S. Circadian variation in platelet function in healthy volunteers. Am J Cardiol 1992;69:951-954.

27. Tofler GH, Brezinski D, Schafer AI, et al. Concurrent morning increase in platelet aggregability and the risk of myocardial infarction and sudden cardiac death. $\mathrm{N}$ Engl J Med 1987;316:1514-1518.

28. Lippi G, Franchini M, Brocco G, Manzato F. Influence of the $\mathrm{ABO}$ blood type on the platelet function analyzer PFA-100. Thromb Haemost 2001;85:369-370.

29. Feuring M, Harenberg J, Peiter A, et al. Impact of ABO blood groups on tirofiban mediated inhibition of platelet function. Platelets 2005;16:430-434. 